ANNALES

POLONICI MATHEMATICI

$83.1(2004)$

\title{
Arc-analyticity and polynomial arcs
}

\author{
by RÉmi Soufflet (Kraków)
}

\begin{abstract}
We relate the notion of arc-analyticity and the one of analyticity on restriction to polynomial arcs and we prove that in the subanalytic setting, these two notions coincide.
\end{abstract}

1. Introduction. In this note, we study some properties of real functions called arc-analytic functions. They are functions defined on an open set $U \subset \mathbb{R}^{n}$ which are analytic on restriction to any analytic arc $\gamma:(-\varepsilon, \varepsilon) \rightarrow U$. Such functions were first introduced by Kurdyka [Ku1] in relation to arcwise symmetric semialgebraic sets.

In general, these functions are very far from being analytic. Several authors have built arc-analytic functions which

- are not continuous (see $[\mathrm{BMP}])$;

- are not subanalytic (see [BMP] and [Ku2]);

- have a non-discrete singular set (see [Ku3]).

It is therefore natural to deal first with subanalytic (or semialgebraic) arc-analytic functions. From recent works of Bierstone and Milman [BM2] and Parusiński [Pa], we know that arc-analytic functions with subanalytic graphs are closely related to the so-called blow-analytic functions introduced by Kuo [Kuo]. The latter are functions which become analytic after finitely many compositions with suitable proper bimeromorphic maps. They give rise to blow-analytic equivalence, a notion which has been studied by many authors (for a general overview of the theory, see $[\mathrm{FKK}]$ ) and which is challenging for the understanding of real-analytic singularities.

The aim of this work is to give a criterion for arc-analyticity (or equivalently local blow-analyticity) which is in some sense algebraic. We will actually prove that it suffices to check the analyticity on curves which are

2000 Mathematics Subject Classification: 32B20, 14P15.

Key words and phrases: subanalytic, arc-analytic, blow-analytic.

Financially supported and member of RAAG network (european program HPRN-CT00271). 
parametrized by polynomials. This gives an easier way to check the local blow-analyticity of a given function. We will use only basic arguments such as the Łojasiewicz inequality.

The author would like to thank J.-M. Lion, W. Pawłucki, J. Stasica and K. Nowak for useful discussions and remarks.

2. Notation and results. In the following, $U$ will always denote an open subset of $\mathbb{R}^{n}$. A polynomial arc $\gamma:(a, b) \rightarrow U,-\infty \leq a<b \leq \infty$, is a mapping such that all its coordinate functions are polynomial functions. The arc $\gamma$ is said to be of degree $k$ if $k$ is the maximum of the degrees of its coordinate functions.

By a subanalytic function, we will always mean a function whose graph is a subanalytic subset of some real projective space (a globally subanalytic subset). We refer to [BM1] for the theory of subanalytic sets.

Even though we will not use the concept of locally blow-analytic functions, we recall their definition for the convenience of the reader (following the definitions given in [BM2] and [Pa]).

Definition 2.1. Let $M$ be a smooth real-analytic manifold. A function $f: M \rightarrow \mathbb{R}$ is called locally blow-analytic if there is a locally finite family of analytic morphisms $\left\{\pi_{j}: M_{j} \rightarrow M\right\}$ and compact sets $K_{j} \subset M_{j}$ such that:

(i) $\bigcup_{j} \pi_{j}\left(K_{j}\right)=M$;

(ii) each $\pi_{j}$ is a composition of finitely many local blowings-up with smooth centers;

(iii) each $f \circ \pi_{j}$ is analytic.

Our criterion of arc-analyticity is based on the following notions of continuity and analyticity on polynomial arcs.

Definition 2.2. Let $f: U \rightarrow \mathbb{R}$. We say that $f$ is

- $\mathcal{P}$-continuous if for all polynomial arcs $\gamma:(-\varepsilon, \varepsilon) \rightarrow U, \varepsilon>0$, the function $f \circ \gamma$ is continuous on $(-\varepsilon, \varepsilon)$;

- $\mathcal{P}$-analytic if for all polynomial arcs $\gamma:(-\varepsilon, \varepsilon) \rightarrow U, \varepsilon>0$, the function $f \circ \gamma$ is analytic on $(-\varepsilon, \varepsilon)$.

The main result says that in the subanalytic setting, the notions of arcanalyticity and $\mathcal{P}$-analyticity coincide. More precisely we have:

Theorem 2.1. Let $f: U \rightarrow \mathbb{R}$ be a subanalytic function. The following conditions are equivalent:

(i) $f$ is $\mathcal{P}$-analytic;

(ii) $f$ is arc-analytic;

(iii) $f$ is locally blow-analytic. 
The equivalence between (ii) and (iii) is a result of Bierstone and Milman (see [BM2] and also [Pa]). We show here that (i) implies (ii). Actually, the main point in this proof is to show that for subanalytic functions, $\mathcal{P}$ continuity implies continuity (Proposition 3.1). The proof of Theorem 2.1 is given in the next section. We finish with some examples and some open questions.

3. Proof of Theorem 2.1. We begin with the following elementary lemma. It is a well known polynomial curve selection lemma for open subanalytic subsets. To make the paper self-contained, we give a proof.

Lemma 3.1. Let $A \subset \mathbb{R}^{n}$ be a subanalytic set of pure dimension $n$ and let $x_{0}$ be a point in the frontier of $A$. Then there exists a polynomial arc $\gamma:(-\varepsilon, \varepsilon) \rightarrow \mathbb{R}^{n}$ such that $\gamma(0)=x_{0}$ and $\gamma((0, \varepsilon)) \subset A$.

Proof. Assume for simplicity that $x_{0}=0$ is the origin of $\mathbb{R}^{n}$. By the usual curve selection lemma for subanalytic subsets, there exists an analytic arc $\mu:(-\varepsilon, \varepsilon) \rightarrow \mathbb{R}^{n}$ such that $\mu(0)=0$ and $\mu((0, \varepsilon)) \subset A$. Denote by $B$ the frontier of $A$. From the Eojasiewicz inequality and up to taking a smaller $\varepsilon>0$, there exist $c>0$ and $k \in \mathbb{N}$ such that, for all $t \in(0, \varepsilon)$, we have

$$
d(B, \mu(t)) \geq c t^{k} .
$$

Let $\mu_{l}$ be the polynomial arc obtained by taking the Taylor expansion of order $l$ of each coordinate function of $\mu$. Fix $l>k$. By the analyticity of $\mu$ there exists a constant $K>0$ such that, for all $t \in(0, \varepsilon)$,

$$
d\left(\mu_{l}(t), \mu(t)\right) \leq K t^{l} .
$$

Assume that $\varepsilon$ is so small that $K t^{l}<c t^{k}$ for all $t \in(0, \varepsilon)$. Then, as $A$ is of pure dimension $n$, the set

$$
C=\bigcup_{t \in(0, \varepsilon)} B\left(\mu(t), K t^{l}\right)
$$

is open and contained in $A$, where $B\left(\mu(t), K t^{l}\right)$ denotes the open ball centered at $\mu(t)$ of radius $K t^{l}$. Then, for $\varepsilon$ yet smaller if necessary, the polynomial arc $\mu_{l+1}:(-\varepsilon, \varepsilon) \rightarrow \mathbb{R}^{n}$ is such that $\mu_{l+1}((0, \varepsilon))$ is contained in $C$. This completes the proof.

The following corollary will also be useful in what follows.

Corollary 3.1. Let $A \subset \mathbb{R}^{n}$ be a subanalytic set of pure dimension $n$ such that

(i) $A=A_{+} \cup A_{-}$, where $A_{+}$and $A_{-}$are open subanalytic sets and $\bar{A}_{+} \cap \bar{A}_{-}=\left\{x_{0}\right\}$;

(ii) there exists an analytic curve $\mu:(-\varepsilon, \varepsilon) \rightarrow \mathbb{R}^{n}$ such that $\mu(0)=x_{0}$, $\mu((-\varepsilon, 0)) \subset A_{-}$and $\mu((0, \varepsilon)) \subset A_{+}$. 
Then there exists a polynomial curve $\gamma:(-\varepsilon, \varepsilon) \rightarrow \mathbb{R}^{n}$ such that $\gamma(0)=$ $x_{0}, \gamma((-\varepsilon, 0)) \subset A_{-}$and $\gamma((0, \varepsilon)) \subset A_{+}$.

Proof. This is immediate from the proof of the previous lemma: it suffices to truncate the asymptotic expansion of $\mu$ both for $t>0$ and for $t<0$, and then to choose the truncation which gives the required inclusions for both $A_{-}$and $A_{+}$.

REMARK 3.1. Note that the corollary is obviously false if we remove the second assumption, as one can see by taking $A_{-}=\left\{(x, y) \in \mathbb{R}_{-}^{2} \mid 3 x<y<\right.$ $2 x<0\}$ and $A_{+}=\left\{(x, y) \in \mathbb{R}_{+}^{2} \mid 0<x<y<2 x\right\}$.

The following lemma is also elementary. It will be used in the proof of Proposition 3.1.

Lemma 3.2. Let $A \subset \mathbb{R}^{n}$ be a subanalytic subset of dimension $d<n$. Then, for all $x \in A$, there exists a linear arc $L:(-\varepsilon, \varepsilon) \rightarrow \mathbb{R}^{n}$ such that $L(0)=x$ and $L((0, \varepsilon)) \subset \mathbb{R}^{n} \backslash A$.

Proof. Assume that there exists $x \in A$ such that for all linear $\operatorname{arcs} L$ passing through $x$, the set $A$ contains an open segment of $L$ containing $x$. Denote by $S_{L}$ such a segment of maximum length. Then the map

$$
D: \mathbb{R} P(n-1) \rightarrow \mathbb{R}
$$

which associates to $L$ the length of $S_{L}$, is subanalytic. It follows that there exists a closed subanalytic subset $Z$ of $\mathbb{R} P(n-1)$ of dimension at most $n-2$ such that $D$ is continuous on $\mathbb{R} P(n-1) \backslash Z$. Let $K \subset \mathbb{R} P(n-1) \backslash Z$ be a compact subset with non-empty interior. The function $D$ is bounded from below on $K$ by a strictly positive constant. This allows us to deduce that the set $A$ must contain a cone of vertex $x$ generated by the lines $L$ belonging to $K$. This contradicts the assumption that $\operatorname{dim}(A)<n$.

The next result is the key point in the proof of Theorem 2.1. It comes from a weaker version due to Bierstone and Milman (see [BM2, Lemma 6.8]).

Proposition 3.1. Let $f: U \rightarrow \mathbb{R}$ be a subanalytic function. Then $f$ is continuous if and only if $f$ is $\mathcal{P}$-continuous.

Proof. As $f$ is subanalytic, it is enough to check the continuity on analytic arcs (see [BM2, Lemma 6.8]). The problem is local so assume that $f(0)=0$ and that $f$ is not continuous at the origin of $\mathbb{R}^{n}$. Then there exist a constant $c>0$ and an analytic arc $\gamma:(-\varepsilon, \varepsilon) \rightarrow \mathbb{R}^{n}$ such that $\gamma(0)=0$ and $|f \circ \gamma(t)| \geq 2 c / 3>0$ for all $t \in(0, \varepsilon)$. Set

$$
A=\left\{x \in \mathbb{R}^{n}|| f(x) \mid \geq c / 3\right\} .
$$

Then $\gamma((0, \varepsilon)) \subset A$. Moreover, we have:

Claim. The subanalytic set $A$ has maximal dimension $n$ at zero. 
Indeed, suppose that $\operatorname{dim}_{0}(A)<n$. Let $t \in(0, \varepsilon)$ and let $B$ be the ball of center $\gamma(t)$ and of radius small enough such that $B$ does not contain the origin. Set

$$
C=B \backslash A \text {. }
$$

By Lemma 3.2, there exists a linear arc $L:(-\delta, \delta) \rightarrow \mathbb{R}^{n}$ such that $L(0)=$ $\gamma(t)$ and $L((0, \delta)) \subset C$. Thus $|f \circ L(s)|<c / 3$ if $s \in(0, \delta)$ and $|f \circ L(0)| \geq$ $2 c / 3$, which contradicts the continuity of $f$ on polynomial arcs. This proves the claim.

It follows from the proof of the claim that there exists an open subanalytic set $E$ such that $E \subset A$ and the origin belongs to the frontier of $E$. We now apply Lemma 3.1: there exists a polynomial arc $\lambda:(-\delta, \delta) \rightarrow \mathbb{R}^{n}$ such that $\lambda((0, \delta)) \subset E$ and $\lambda(0)=0$. Then $|f \circ \lambda(s)| \geq c / 3$ for all $s \in(0, \delta)$ and $f \circ \lambda(0)=0$. Thus $f$ restricted to $\lambda$ is not continuous and the proof is complete.

Proof of Theorem 2.1. Suppose that $f$ is $\mathcal{P}$-analytic but not arc-analytic. Then there exists an analytic arc $\gamma:(-\varepsilon, \varepsilon) \rightarrow \mathbb{R}^{n}$ such that $g=f \circ \gamma$ is not analytic at the origin (up to translation, this can always be assumed). Two cases arise:

CAse 1. As $g$ is subanalytic, it has a Puiseux expansion for $t \in(0, \varepsilon)$ :

$$
g(t)=\sum_{i \geq 0} a_{i} t^{n_{i} / q}
$$

with $q \geq 1$. In this first case, we assume that this expansion is not the expansion of an analytic function at 0 (i.e. $q>1$ ). We then write it in the form

$$
g(t)=p(t)+a t^{r}+b t^{s}+o_{0}\left(t^{s}\right)
$$

where $p$ is a polynomial, $r$ is the smallest exponent which is not an integer and $s>r$.

Now consider the following set:

$$
A=\left\{(t, v) \in(0, \varepsilon) \times \mathbb{R}|| v-g(t) \mid<t^{s}\right\} .
$$

Then $A$ is an open subanalytic set containing $\Gamma=\{(t, g(t)) \mid t \in(0, \varepsilon)\}$. The map $F:(0, \varepsilon) \times \mathbb{R}^{n} \rightarrow \mathbb{R}^{2}$ defined by $F(t, u)=(t, f(u))$ is subanalytic and continuous by Proposition 3.1. Let $G=\{(t, \gamma(t)) \mid t \in(0, \varepsilon)\}$. Then $B=F^{-1}(A)$ is an open subanalytic set containing $G$. From the proof of Lemma 3.1, there exists a truncation of $\gamma$, which is a polynomial arc $\mu$ : $(-\varepsilon, \varepsilon) \rightarrow \mathbb{R}^{n}$, such that $\mu(0)=0$ and the graph of $\mu$ lies in $B$ for $t \in(0, \varepsilon)$. Then, for all $t \in(0, \varepsilon)$, we have

$$
|f \circ \mu(t)-g(t)|<t^{s}
$$


and so $f \circ \mu$ has the same asymptotic expansion as $g$ up to order $r$. This contradicts the analyticity of $f$ restricted to the polynomial arc $\mu$.

CASE 2. Denote by $g_{-}$and $g_{+}$the asymptotic expansions of $f \circ \gamma$ on $(-\varepsilon, 0)$ and $(0, \varepsilon)$ respectively. In this second case, we assume that both $g_{-}$ and $g_{+}$extend to analytic functions in a neighborhood of 0 but $g_{-} \neq g_{+}$. Write

$$
\begin{aligned}
& g_{-}(t)=\sum_{n \geq 0} a_{n}^{-} t^{n}, \quad t \in(-\varepsilon, 0), \\
& g_{+}(t)=\sum_{n \geq 0} a_{n}^{+} t^{n}, \quad t \in(0, \varepsilon),
\end{aligned}
$$

and let $p>0$ be the first integer such that $a_{p}^{-} \neq a_{p}^{+}$. As in the previous case, we now consider the sets

$$
\begin{aligned}
& A_{-}=\left\{(t, v) \in(-\varepsilon, 0) \times \mathbb{R}|| v-g_{-}(t) \mid<t^{p+1}\right\}, \\
& A_{+}=\left\{(t, v) \in(0, \varepsilon) \times \mathbb{R}|| v-g_{+}(t) \mid<t^{p+1}\right\},
\end{aligned}
$$

and the sets $B_{-}=F^{-1}\left(A_{-}\right), B_{+}=F^{-1}\left(A_{+}\right)$and $B=B_{-} \cup B_{+}$for the same map $F$ as in the preceding case. These sets are open and satisfy the assumption of Corollary 3.1 for the analytic arc $\gamma$. Hence one can find a polynomial arc (once more a truncation of $\gamma) \delta:(-\varepsilon, \varepsilon) \rightarrow \mathbb{R}^{n}$ such that $\delta((-\varepsilon, 0)) \subset B_{-}$and $\delta((0, \varepsilon)) \subset B_{+}$. This implies that, for all $t \in(-\varepsilon, \varepsilon) \backslash\{0\}$,

$$
\left|f \circ \delta(t)-g_{ \pm}(t)\right|<t^{p+1} \text {. }
$$

Thus $f \circ \delta$ has the same asymptotic expansion as $g_{-}$and $g_{+}$up to order $p$, which gives a contradiction.

REMARK 3.2. The analyticity on restriction to polynomial arcs with degrees bounded by some constant $k \in \mathbb{N}$ does not imply the arc-analyticity for all subanalytic functions. Actually such a $k$ depends on each subanalytic function considered. Take for instance

$$
f(x, y)=\frac{x y^{2}}{x^{2}+y^{4}}, \quad(x, y) \neq(0,0),
$$

and $f(0)=0$. Then $f$ is a subanalytic function which is analytic when restricted to any (affine) line in $\mathbb{R}^{2}$ but which is not arc-analytic. This way the reader can build examples of subanalytic functions which are analytic on restriction to polynomial arcs with degree less than $k$ but which are not arc-analytic (for all $k$ ).

Remark 3.3. Proposition 3.1 is not true in general for non-subanalytic functions. Consider for instance the function

$$
f(x, y)=\frac{|x|^{\lambda} y}{|x|^{2 \lambda}+y^{2}}, \quad(x, y) \neq(0,0),
$$


such that $f(0)=0$ and $0<\lambda<1$ is irrational. Then it is easy to check that $f$ is continuous on every analytic arc but not on the arc given by $y=|x|^{\lambda}$.

This example shows that there is no straightforward generalization of Proposition 3.1 (or Theorem 2.1) if we replace "subanalytic" by "definable in some o-minimal structure" (even polynomially bounded, see [DM]).

REMARK 3.4. A pointwise version of Proposition 3.1 should exist but with some further assumption. Indeed, $\mathcal{P}$-continuity at one point $x_{0}$ (i.e. continuity on restriction to germs of polynomial arcs passing through the given point $x_{0}$ ) does not imply continuity at this point even in the algebraic case as one can see from the following example: let $f$ be the function defined by

$$
f(x, y)=1 \quad \text { if } x<0 \text { and } y^{2}=x\left(x^{2}-1\right),
$$

and $f(x, y)=0$ if not. Then $f$ is $\mathcal{P}$-continuous at the origin but of course not continuous at the origin. This follows from the fact that the cubic curve $y^{2}=x\left(x^{2}-1\right)$ is not rational (or unicursal) and thus cannot be parametrized by polynomial functions (its genus is not equal to zero).

Questions and remarks. The notions of arc-analyticity and $\mathcal{P}$-analyticity can be defined at points in the following way:

Definition 3.1. Let $f: U \rightarrow \mathbb{R}$ be a function and $x \in U$. We say that $f$ is arc-analytic (resp. $\mathcal{P}$-analytic) at $x$ if it is analytic on restriction to any germ of analytic (resp. polynomial) curve passing through $x$.

From a recent work of Kurdyka and Paunescu, we know that if $f$ is a continuous bounded subanalytic function, then the set $S$ of points where it is not arc-analytic is a subanalytic closed and nowhere dense subset $[\mathrm{KP}]$. In this paper, we have proved the following two facts (for $f$ subanalytic):

(i) If $f$ is $\mathcal{P}$-continuous at $x_{0}$ and continuous on segments in a neighborhood of $x_{0}$ then $f$ is continuous at $x_{0}$.

(ii) If $f$ is $\mathcal{P}$-analytic at $x_{0}$ and continuous in a neighborhood of $x_{0}$ then $f$ is arc-analytic at $x_{0}$.

Hence by $[\mathrm{KP}]$ and the second statement (corresponding to Theorem 2.1), for bounded continuous subanalytic functions, the notion of arc-analyticity at a given point coincides with the one of $\mathcal{P}$-analyticity at this point. Therefore, the following questions seem to be of interest:

1 (Uniform version of Theorem 2.1). Let $f: U \rightarrow \mathbb{R}$ be a continuous bounded subanalytic function. Does there exist $k \in \mathbb{N}$ such that, for all $x_{0} \in U$, if $f$ is analytic on restriction to germs of polynomial arcs of degree at most $k$ passing through $x_{0}$, then $f$ is arc-analytic at $x_{0}\left({ }^{1}\right)$ ?

$\left({ }^{1}\right)$ The author has recently proved that the answer is yes. This will be presented in another paper. 
2. Let $d, n$ be some integers. Does there exist $k(d, n) \in \mathbb{N}$ such that, for all polynomials $P \in \mathbb{R}\left[x_{1}, \ldots, x_{n}, y\right]$ of degree at most $d$ with $P(0)=0$, and for all roots $f: U \rightarrow \mathbb{R}$ of $P$ (i.e. $P(x, f(x))=0$ for all $x \in U, U$ a neighborhood of the origin in $\mathbb{R}^{n}$ ), if $f$ is analytic on restriction to germs of polynomial arcs of degree at most $k(d, n)$ passing through 0 , then $f$ is arc-analytic at 0 ?

If we drop the assumption of continuity, the answer to the question in item 1 is negative, as one can see from Example 3.4 or from the following example: let $f: \mathbb{R}^{2} \rightarrow \mathbb{R}$ be defined by $f(x, y)=1$ if $(x, y)=\left(\sin ^{2}(t), t^{3}\right)$ and $t>0$, and $f(x, y)=0$ if not.

\section{References}

[BM1] E. Bierstone and P. D. Milman, Semianalytic and subanalytic sets, Publ. Math. IHES 67 (1988), 5-42.

[BM2] -, -, Arc-analytic functions, Invent. Math. 101 (1990), 411-424.

[BMP] E. Bierstone, P. D. Milman and A. Parusiński, A function which is arc-analytic but not continuous, Proc. Amer. Math. Soc. 113 (1991), 419-424.

[DM] L. van den Dries and C. Miller, Geometric categories and o-minimal structure, Duke Math. J. 84 (1996), 497-540.

[FKK] T. Fukui, S. Koike and T.-C. Kuo, Blow-analytic equisingularities, properties, problems and progress, in: Real Analytic and Algebraic Singularities, T. Fukuda et al. (eds.), Pitman Res. Notes Math. Ser. 381, Longman, 1998, 8-29.

[Kuo] T. -C. Kuo, On classification of real singularities, Invent. Math. 82 (1985), 257262.

[Ku1] K. Kurdyka, Ensembles semi-algébriques symétriques par arcs, Math. Ann. 281 (1988), 445-462.

[Ku2] -, A counterexample to subanalyticity of an arc-analytic function, Ann. Polon. Math. 55 (1991), 241-243.

[Ku3] -, An arc-analytic function with non-discrete singular set, ibid. 59 (1994), 251254.

[KP] K. Kurdyka and L. Paunescu, Arc-analyticity is an open property, preprint, 2003.

[Pa] A. Parusiński, Subanalytic functions, Trans. Amer. Math. Soc. 344 (1994), 583595.

Institute of Mathematics

Jagiellonian University

Reymonta 4

30-059 Kraków, Poland

E-mail: remi.soufflet@u-bourgogne.fr

Reçu par la Rédaction le 23.4.2003

Révisé le 5.12.2003 